\title{
Search strategies for charged Higgs bosons in ATLAS
}

\author{
Arnaud FERRARI* on behalf of the ATLAS collaboration \\ Uppsala University, Sweden \\ E-mail: arnaud.ferrariefysast.uu.se
}

Charged Higgs bosons are predicted by various theories beyond the Standard Model, where the Higgs sector does not only consist of one doublet of scalar fields. Search strategies for charged Higgs bosons in the ATLAS experiment at the Large Hadron Collider (LHC) are presented. In particular, if $m_{H^{+}}<m_{t o p}$, the top quark can decay to a bottom quark and a light charged Higgs boson, for which some of the dominant decays are $H^{+} \rightarrow c \bar{s}$ and $H^{+} \rightarrow \tau^{+} v$ (depending on the parameter space). After a reminder of the ATLAS search strategies for heavy charged Higgs bosons, for a $14 \mathrm{TeV}$ LHC operation, results of Monte-Carlo studies recently performed for a $7 \mathrm{TeV}$ centre-of-mass energy, i.e. for the early data-taking period, are presented.

Prospects for Charged Higgs Discovery at Colliders

27-30 September 2010

Uppsala University, Sweden

\footnotetext{
*Speaker.
} 


\section{Introduction}

One major task for the Large Hadron Collider (LHC) and the ATLAS experiment will be the exploration of the Higgs sector, in the Standard Model (SM) and beyond it [1]. Several extensions of the SM, so-called Two-Higgs Doublets Models (2HDM), such as the Minimal Supersymmetric extension of the Standard Model (MSSM), require at least two isodoublets of scalar fields in the Higgs sector. Electroweak symmetry breaking through two complex Higgs doublets lead to five physical states, out of which two are charged $\left(\mathrm{H}^{+}\right.$and $\left.\mathrm{H}^{-}\right)$. A priori, the mass of the charged Higgs bosons is not predicted by theory. However, at tree level, the MSSM Higgs sector is fully determined by two parameters: $m_{H^{ \pm}}$and the ratio of the two Higgs doublet vacuum expectation values $\tan \beta$. Note that some extensions of the SM, based on the Higgs Triplet Model, predict (doubly) charged Higgs, but those are not addressed in this paper.

The discovery of a charged Higgs boson would undoubtedly prove the existence of new physics beyond the SM. Search strategies depend on the hypothesized mass of the charged Higgs boson, since it determines both its production and the decay modes. This note first presents the ATLAS discovery potential for a heavy $H^{ \pm}$(close to or beyond the top quark mass) when the LHC will operate at full energy $(14 \mathrm{TeV})$. On the other hand, if $H^{ \pm}$is lighter than the top quark, it can be copiously produced in $t \bar{t}$ pairs during the early LHC data-taking period, even with a centre-of-mass energy of $7 \mathrm{TeV}$. Search strategies for light charged Higgs bosons in ATLAS will therefore be discussed in detail.

\section{ATLAS search strategies for heavy charged Higgs bosons in $14 \mathrm{TeV}$ pp collisions}

If the charged Higgs boson is heavier than the top quark, then it is dominantly produced via $g g \rightarrow t b H^{+}$or $g b \rightarrow t H^{+}$at the LHC, with cross sections in the pb range ${ }^{1}$. The dominant decay channel is $H^{+} \rightarrow t \bar{b}$ (with a branching ratio above 90\%, depending on the values of $m_{H^{+}}$and $\tan \beta$ ). Still, $H^{+} \rightarrow \tau^{+} v$ remains sizeable and offers a significantly cleaner signature. Discovery prospects for heavy charged Higgs bosons in ATLAS with a $14 \mathrm{TeV}$ LHC centre-of-energy and an integrated luminosity in the $1-30 \mathrm{fb}^{-1}$ range were previously discussed in e.g. [2] and [3]: only a summary of the main results is given here.

The most promising channels are $g g \rightarrow t b H^{+} \rightarrow b q q b \tau^{+} v$ and $g b \rightarrow t H^{+} \rightarrow b q q \tau^{+} v$, for which the event selection consists of a hard $\tau$ jet, large missing $E_{T}$, one or two $b$-tagged jets, as well as a hadronic $W$ boson, which can be fully reconstructed and combined with a $b$-tagged jet in order to form a top quark. The background is dominated by $t \bar{t}$ and it can be reduced by using an uncorrelated likelihood approach with five variables: $p_{T}(\tau), E_{T}^{\text {miss }}$, the azimuthal angle $\Delta \phi\left(\tau, E_{T}^{\text {miss }}\right)$, $\sum_{j \neq \tau} p_{T}(j)$, and the $p_{T}$ ratio between the $\tau$ jet and the hardest jet not coming from the top quark. The discovery prospect for this channel covers a region with $m_{H^{+}}$up to $350 \mathrm{GeV}$ and high values of $\tan \beta$.

\footnotetext{
${ }^{1}$ In the following, the charged Higgs bosons will only be denoted as $H^{+}$but charge conjugated processes are always implicitly included.
} 
The other channels investigated by ATLAS for the discovery of heavy charged Higgs bosons are $g g \rightarrow t b H^{+} \rightarrow b \ell v b b q q b$ and $g b \rightarrow t H^{+} \rightarrow b \ell v b q q b$, for which the event selection is based on an isolated lepton and at least 5 jets (three of them $b$-tagged), with a successful reconstruction of a leptonic $W$ boson. A combinatorial likelihood is first used in order to suppress the combinatorial background, followed by a selection likelihood aimed at reducing the physical background (which is dominated by $t \bar{t}+$ jets events). Although this channel does not offer a discovery potential for $\mathrm{H}^{+}$ in ATLAS on its own, it contributes to the combined sensitivity and may allow to measure $m_{H^{+}}$ upon discovery of the charged Higgs boson using another, more sensitive, channel.

Figure 1 shows the ATLAS discovery and exclusion sensitivity contours for a heavy $\mathrm{H}^{+}$in $p p$ collisions at $14 \mathrm{TeV}$, in a model-independent way, i.e. as a function of $m_{H^{+}}$and of the production cross section for $H^{+} \rightarrow \tau^{+} v$ in $g g$ or $g b$ fusion. A total systematic uncertainty of $10 \%$ is assumed for the $t \bar{t}$ background. As for the signal, the dominant theoretical and experimental uncertainties (dominated by the jet energy scale) reach $20 \%$ to $40 \%$, depending on the channel used for $\mathrm{H}^{+}$ discovery/exclusion.
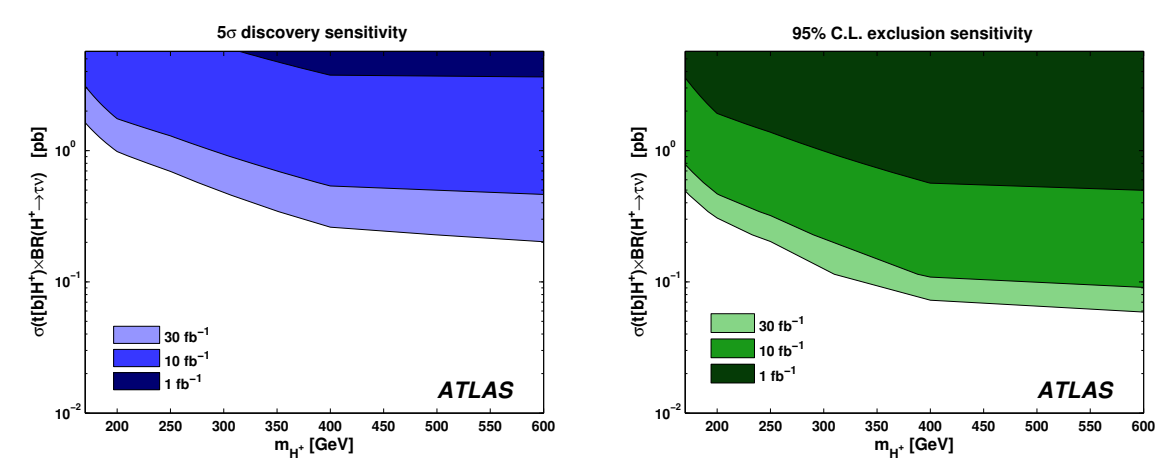

Figure 1: ATLAS combined discovery and exclusion sensitivities for heavy charged Higgs bosons, in terms of the production cross section for $H^{+} \rightarrow \tau^{+} v$ in $g g$ or $g b$ fusion.

\section{ATLAS search strategies for light charged Higgs bosons in $7 \mathrm{TeV}$ pp collisions}

If $H^{+}$is lighter than the top quark, it can appear in $t \rightarrow b H^{+}$and thus be searched for in the cascade decays of $t \bar{t}$ pairs, which will be copiously produced at LHC, via $g g$ or $q \bar{q}$ fusion. Here, we summarize two sensitivity studies for light charged Higgs bosons, with an integrated luminosity of $1 \mathrm{fb}^{-1}$ and $\sqrt{s}=7 \mathrm{TeV}$, in semi-leptonic and di-lepton $t \bar{t}$ events [4].

\section{1 $H^{+} \rightarrow c \bar{s}$ in semi-leptonic $t \bar{t}$ events}

For $\tan \beta<1$, the branching ratio $\mathscr{B}\left(H^{+} \rightarrow c \bar{s}\right)$ may reach $40 \%$ for a charged Higgs boson mass close to $130 \mathrm{GeV}$ in the MSSM, making this decay channel a viable one for charged Higgs boson searches in ATLAS. For this purpose, a pre-selection for semi-leptonic $t \bar{t}$ events is first performed. Events passing a high $p_{T}$ lepton trigger are required to have an electron or a muon with $p_{T}>20 \mathrm{GeV}$ within $|\eta|<2.5$, some missing transverse energy larger than $20 \mathrm{GeV}$, and the four 
leading jets (with two $b$-tagged jets) must have $p_{T}>20 \mathrm{GeV}$ and be within $|\eta|<2.5$. The largest background comes from $W$ bosons in SM $t \bar{t}$ events (95\% of the total background). In the preselected events, the mass of the $H^{+} \rightarrow c \bar{s}$ candidate (or of the hadronic $W$ boson in the background events) is directly reconstructed using the two untagged jets. The resolution of the resulting di-jet mass distributions can be improved by using a kinematical fitter based on the full reconstruction of the leptonic $W$ boson and the two top quarks, where the transverse energies of the final state objects (lepton, jets) are allowed to vary within measurement uncertainties, see Figure 2.
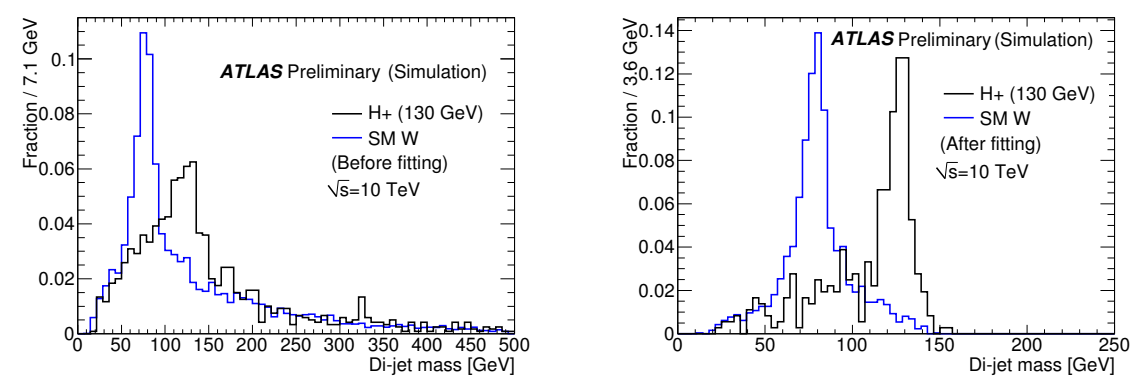

Figure 2: Left: the normalised di-jet mass templates directly reconstructed from the two untagged jets. Right: the improved, normalised di-jet mass templates using the kinematical fitter.

Assuming that we find no signal beyond the SM, a binned maximum likelihood fit is applied on the di-jet mass distribution to compute the upper limit at $95 \%$ confidence level for the branching ratio $\mathscr{B}\left(t \rightarrow b H^{+}\right)$. In the fit, $\mathscr{B}\left(t \rightarrow b H^{+}\right)$and the total number of $t \bar{t}$ events are free parameters, while the amount of non- $t \bar{t}$ background has a Gaussian constraint, $67 \pm 20$ events for an integrated luminosity of $1 \mathrm{fb}^{-1}$. The upper limit on $\mathscr{B}\left(t \rightarrow b H^{+}\right)$at $95 \%$ confidence level is extracted using pseudo-experiments, assuming $\mathscr{B}\left(H^{+} \rightarrow c \bar{s}\right)=1$ and taking into account both statistical and systematic uncertainties, the latter being represented by a nuisance parameter in the likelihood, that adds to the branching ratio $\mathscr{B}$ and has a Gaussian prior probability density function with a width $\triangle \mathscr{B}$. We eliminate this nuisance parameter by Bayesian marginalisation and obtain a posterior probability density function in $\mathscr{B}$, assuming a uniform prior in $0<\mathscr{B}\left(t \rightarrow b H^{+}\right)<1$. Note that, in this analysis, the jet energy scale is calibrated by using the peak position of the unfitted di-jet mass distribution, hence the corresponding systematic error is very small.

The expected upper limits on $\mathscr{B}\left(t \rightarrow b H^{+}\right)$at $95 \%$ confidence level, for $H^{+}$masses between 90 to $150 \mathrm{GeV}$, are shown in Table 1. The expected limits in the second row are based on the statistical errors only, while the expected limits in the third row include both statistical and systematic errors.

\begin{tabular}{|c|cccc|}
\hline$m_{H^{+}}(\mathrm{GeV})$ & 90 & 110 & 130 & 150 \\
\hline Expected upper limit $\mathscr{B}\left(t \rightarrow b H^{+}\right)$(stat. only) & $4.0 \%$ & $2.5 \%$ & $2.3 \%$ & $1.5 \%$ \\
Expected upper limit $\mathscr{B}\left(t \rightarrow b H^{+}\right)$(stat + syst) & $14.8 \%$ & $4.7 \%$ & $3.4 \%$ & $3.7 \%$ \\
\hline
\end{tabular}

Table 1: Expected upper limits on $\mathscr{B}\left(t \rightarrow b H^{+}\right)$at $95 \%$ confidence level, for $H^{+}$masses between 90 to $150 \mathrm{GeV}$, for an integrated luminosity of $1 \mathrm{fb}^{-1}$ at $7 \mathrm{TeV}$. 


\section{2 $H^{+} \rightarrow \tau_{l e p} v$ in di-lepton $t \bar{t}$ events}

In the MSSM, for $\tan \beta>1, H^{+} \rightarrow \tau^{+} v$ dominates other decays. If $\tan \beta>3, \mathscr{B}\left(H^{+} \rightarrow \tau^{+} v\right)$ exceeds $90 \%$. Here, we only consider leptonic $\tau$ decays and we report on light $H^{+}$search strategies in di-lepton $t \bar{t}$ events. For this purpose, two new tools have been considered [5, 6]:

- $\cos \theta_{l}^{*} \simeq \frac{4 p_{b} \cdot p_{l}}{m_{t}^{2}-m_{W}^{2}}-1$, where $p_{b}$ and $p_{l}$ can be advantageously chosen in the laboratory frame,

- the generalised transverse mass $m_{T 2}^{H^{+}}$, computed using with numerical methods and defined as the maximum of the invariant mass $\left(p^{H^{+}}\right)^{2}$ subjected to the following constraints:

$$
\begin{aligned}
\left(p^{H^{+}}+p^{b}\right)^{2} & =m_{t o p}^{2}, \\
\left(p^{\ell^{-}}+p^{\bar{v}_{\ell}}\right)^{2} & =m_{W}^{2}, \\
\left(p^{\ell^{-}}+p^{\bar{v}_{\ell}}+p^{\bar{b}}\right)^{2} & =m_{t o p}^{2}, \\
\left(p^{\bar{v}_{\ell}}\right)^{2} & =0, \\
\vec{p}_{T}^{H^{+}}-\vec{p}_{T}^{l^{+}}+\vec{p}_{T}^{\bar{v}_{\ell}} & =\vec{p}_{T}^{\text {miss }} .
\end{aligned}
$$

The general selection of di-lepton $t \bar{t}$ candidate events first relies on the positive decision of a high $p_{T}$ lepton trigger. We then require the presence of two oppositely charged leptons (electron or muon) with $|\eta|<2.5$, as well as $p_{T}>20 \mathrm{GeV}$ (leading) and $p_{T}>10 \mathrm{GeV}$ (sub-leading). The remaining events must have at least two jets with $p_{T}>15 \mathrm{GeV}$ and $|\eta|<5$. The two jets with the highest likelihood of being $b$-tagged jets are assumed to be daughters of the top and anti-top quarks. At this stage, the four-fold ambiguity in assigning the leptons and the $b$-jets to their parents is resolved using $\cos \theta_{l}^{*}$ and $m_{T 2}^{H^{+}}$, thereby defining a $W$ side and a $H^{+}$side for each event. We then apply selection cuts on the $b$-weight and $E_{T}^{\text {miss }}$ in order to isolate di-lepton $t \bar{t}$ events. Figure 3 shows the $\cos \theta_{l}^{*}$ distributions on the $H^{+}$side in the SM case (dashed line) or with a $130 \mathrm{GeV}$ charged Higgs boson and $\mathscr{B}\left(t \rightarrow b H^{+}\right)=17 \%$, which is the upper limit from Tevatron at the time of writing. A significant excess of events in the bin closest to -1 can show evidence for the presence of a charged Higgs boson in the top quark decays.

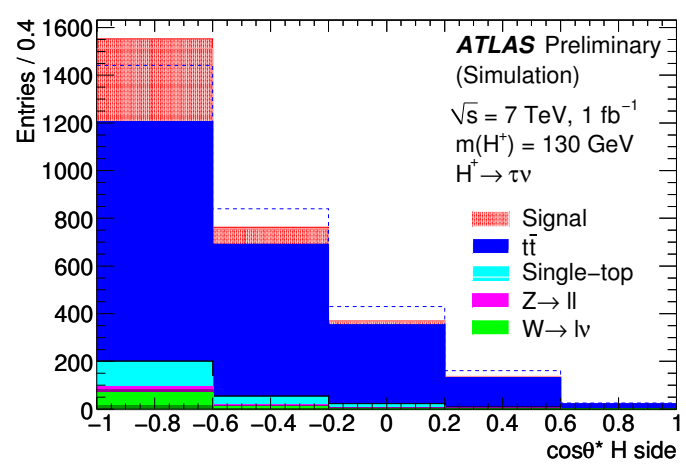

Figure 3: Comparison of the $\cos \theta_{l}^{*}$ distribution on the $H^{+}$side in the SM case (dashed line) and as obtained when assuming $m_{H^{+}}=130 \mathrm{GeV}$ and $\mathscr{B}\left(t \rightarrow b H^{+}\right)=17 \%$ (filled histogram). All selection cuts are applied. 
Assuming that we find no signal beyond the $\mathrm{SM}$, and that $\mathscr{B}\left(H^{+} \rightarrow \tau^{+} v\right)=1$, the upper limit on the branching ratio $\mathscr{B}\left(t \rightarrow b H^{+}\right)$is given by:

$$
\mathscr{B}=\frac{N_{\mathrm{obs}}-N_{\mathrm{bg}}}{2 \times \sigma_{t \bar{t}} \times \mathscr{L}_{\text {int }} \times \varepsilon_{\mathrm{sig}}},
$$

where $N_{\text {obs }}$ is the number of observed events surviving the di-lepton $t \bar{t}$ selection criteria together with $\cos \theta_{l}^{*}<-0.6$ on the $H^{+}$side, with an expectation value $N_{\mathrm{bg}}$ for the background.

We evaluate $\mathscr{B}$ with toy Monte-Carlo experiments, varying the input parameters $N_{\mathrm{obs}}, N_{\mathrm{bg}}$ and $\varepsilon_{\text {sig }}$ by their uncertainties (note that $\sigma_{t \bar{t}} \times \mathscr{L}_{\text {int }}$ is measured from data in a specific sideband). A probability weight for each $\mathscr{B}$ is determined by:

$$
W_{\mathscr{B}}\left(N_{\mathrm{obs}}, N_{\mathrm{bg}}, \varepsilon_{\mathrm{sig}}\right)=P\left(N_{\mathrm{obs}}\right) \times P\left(N_{\mathrm{bg}}\right) \times P\left(\varepsilon_{\mathrm{sig}}\right) .
$$

Here, $P(x)$ are modelled by Gaussian probability density functions. The width of the $N_{\text {obs }}$ distribution is the Poisson uncertainty $\sqrt{N_{\mathrm{obs}}}$, while the widths of $N_{\mathrm{bg}}$ and $\varepsilon_{\text {sig }}$ are determined by Monte-Carlo statistics and systematic uncertainties. The branching ratios $\mathscr{B}$ were evaluated with and without the systematic uncertainties. Each value of $\mathscr{B}$ is multiplied by the corresponding probability weight $W_{\mathscr{B}}$ before solving for the $95 \%$ confidence level upper limit:

$$
0.95=\int_{0}^{\mathscr{B} 95 \%} \mathscr{B} d \mathscr{B} .
$$

The expected upper limits on $\mathscr{B}\left(t \rightarrow b H^{+}\right)$at $95 \%$ confidence level, for $H^{+}$masses between 90 to $150 \mathrm{GeV}$, are shown in Table 2. The expected limits in the second colum are based on the

\begin{tabular}{|c|c|c|}
\hline Mass & \multicolumn{2}{|c|}{ Expected upper limit on $\mathscr{B}\left(t \rightarrow b H^{+}\right)$} \\
\hline$(\mathrm{GeV})$ & without systematics & with systematics \\
\hline 90 & $6.5 \%$ & $8.9 \%$ \\
\hline 110 & $5.6 \%$ & $7.4 \%$ \\
\hline 130 & $5.6 \%$ & $7.4 \%$ \\
\hline 150 & $6.6 \%$ & $8.6 \%$ \\
\hline
\end{tabular}
statistical errors only, while the expected limits in the third column include both statistical and systematic errors.

Table 2: Expected upper limits on $\mathscr{B}\left(t \rightarrow b H^{+}\right)$at $95 \%$ confidence level, for $H^{+}$masses between 90 to $150 \mathrm{GeV}$, for an integrated luminosity of $1 \mathrm{fb}^{-1}$ at $7 \mathrm{TeV}$.

\section{Conclusion}

In this note, ATLAS search strategies for charged Higgs bosons were summarized, focusing first on the high mass range (i.e. when $H^{+}$is produced with an associated top quark via $g g$ or $g b$ fusion) for an integrated luminosity greater than $1 \mathrm{fb}^{-1}$ at $14 \mathrm{TeV}$. We then presented recent ATLAS simulation studies of the production of light charged Higgs bosons in semi-leptonic and di-lepton $t \bar{t}$ events. Assuming separately a $100 \%$ branching ratio for $H^{+} \rightarrow c \bar{s}$ or $H^{+} \rightarrow \tau^{+} \nu$, 
we derived upper limits on $\mathscr{B}\left(t \rightarrow b H^{+}\right)$at $95 \%$ confidence level for an integrated luminosity of $1 \mathrm{fb}^{-1}$ at $7 \mathrm{TeV}$. Those upper limits are summarized graphically in Figure 4, where we also show that ATLAS can substantially improve the upper limits on $\mathscr{B}\left(t \rightarrow b H^{+}\right)$from the Tevatron at the time of writing $[7,8,9]$.
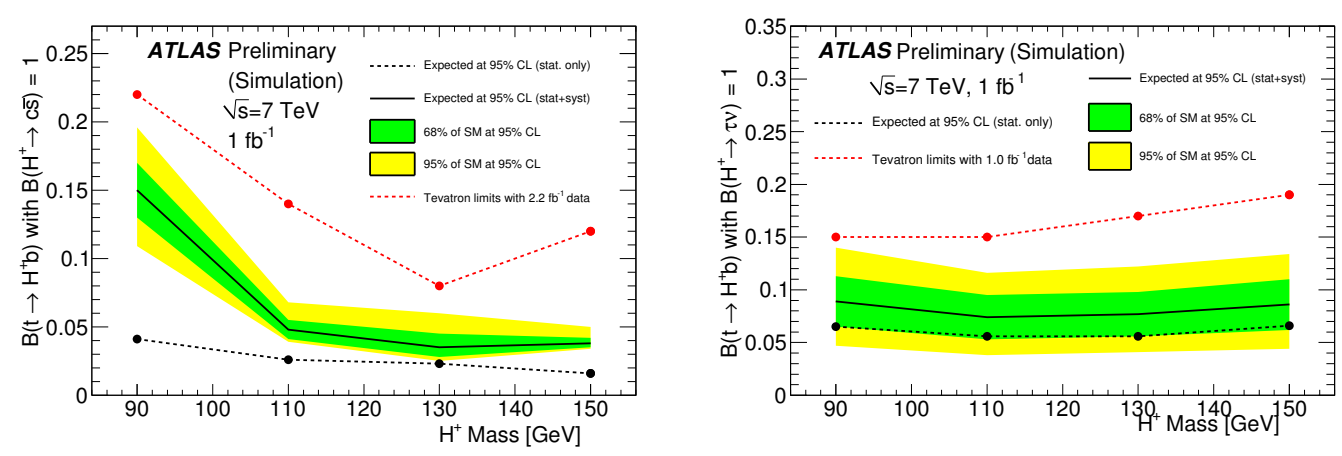

Figure 4: Expected upper limits on $\mathscr{B}\left(t \rightarrow b H^{+}\right)$at $95 \%$ confidence level in early data (i.e. with $1 \mathrm{fb}^{-1}$ at $7 \mathrm{TeV}$ ) versus the charged Higgs boson mass assuming $\mathscr{B}\left(H^{+} \rightarrow c \bar{s}\right)=1$ (left) or $\mathscr{B}\left(H^{+} \rightarrow \tau^{+} v\right)=1$ (right). The green and yellow bands correspond to the range in which we expect the limit to lie, depending upon data.

\section{References}

[1] J.F. Gunion, H.E. Haber, G.L. Kane and S. Dawson, The Higgs Hunter's Guide (Addison-Wesley, Reading, 1990).

[2] ATLAS Collaboration, arXiv:0901.0512 \& CERN-OPEN-2008-020.

[3] M. Flechl, PoS (CHARGED2008) 006.

[4] ATLAS Collaboration, ATL-PHYS-PUB-2010-009.

[5] D. Eriksson, G. Ingelman, J. Rathsman and O. Stål, JHEP 01, 024 (2008).

[6] E. Gross and O. Vitells, [arXiv:0907.5367v1] (2009).

[7] CDF Collaboration, CDF Note 9322 [arXiv:0906.1269] (2009).

[8] D0 Collaboration, D0 Note 5715-CONF (2008).

[9] D0 Collaboration, Phys. Rev. Lett. 102, 191802 (2009). 